\title{
Enhancement of particle collection efficiency considering the structural interplay: particle motion characteristics analysis
}

\author{
Yuan $\mathrm{Xi}^{1,2}$, Yong-liang Zhang ${ }^{3}$, Xi-long Zhang ${ }^{3,}$, and Yan Dai ${ }^{1}$ \\ ${ }^{1}$ Panjin Institute of Industrial Technology, Liaoning Key Laboratory of Chemical Additive Synthesis and Separation, Dalian \\ University of Technology, Panjin 124221, PR China \\ 2 State Key Laboratory of Fluorine Containing Functiona, Shandong Huaxia Shenzhou New Material Co. Ltd., Zibo 255000, \\ PR China \\ ${ }^{3}$ School of Mechanical \& Automotive Engineering, Qingdao University of Technology, Qingdao 266071, PR China
}

Received: 13 August 2020 / Accepted: 25 November 2020

\begin{abstract}
The vacuum-blowing cleaning system, utilizing positive and negative pressure mixed-delivery theory, has been adopted for the road sweeper vehicle. To enhance the solid particle separation performance and to evaluate the motion characteristics of inhaled particles under different structural parameters, the gas-solid flow in the vacuum-blowing cleaning system was investigated by using computational fluid dynamics (CFD) technology. The influence of the main structural parameters on the grade dust collection efficiency and average detention time of the inhaled particles was determined, such as suction-inlet diameter, suction-inlet inclination angle, and front baffle inclination angle. And the interplay between them was also investigated. In addition, a dust collection efficiency model was built, based on uniform design (UD) and multiple regression analysis (MRA), and subsequently verified via experiments. The results revealed that the structural parameters have significant influence on the dust collection performance. The suction-inlet diameter, front baffle inclination angle, and suction-inlet inclination angle exerted the highest, second-highest, and lowest influence, respectively. Furthermore, the interaction among structural parameters also influenced the collection performance. The highest, second-highest, and lowest levels of influence were determined for the inlet diameter/baffle inclination angle, inlet inclination angle/baffle inclination angle, and inlet diameter/inlet inclination interactions, respectively. The highest dust collection efficiency (i.e., 96.10\%) and a short average detention time of particles in the chamber were realized under the following conditions: suction-inlet diameter and inclination angle: $200 \mathrm{~mm}$ and $110^{\circ}$, respectively, and front baffle inclination angle: $105^{\circ}$.
\end{abstract}

Keywords: Road sweeper vehicle / vacuum-blowing cleaning system / CFD / particle motion characteristics / uniform design / multiple regression analysis

\section{Introduction}

In recent years, particulate pollution has become increasingly severe, and has attracted considerable attention [1]. Road dust and sediment give rise to urban particulate pollution. Rapid and efficient removal of these small particles has become essential for building environmentally friendly cities $[2,3]$. The health of the citizens is also impacted by the road particulate matter. According to related medical research reports, the road particulate matter may lead to the death of premature infants [4], respiratory tract infections $[5,6]$, and deprementia $[7,8]$ or other adult-affecting diseases [9-11].

\footnotetext{
* e-mail: zhangxilong@qut.edu.cn
}

A road sweeper vehicle is a sanitation device, that is, a new high-efficiency cleaning device integrating road cleaning, garbage collection, and transportation [12-14]. This device is considered the most efficient for the cleaning of dust, sediment, and rainwater, thereby yielding significant labor-force savings with consideration of the cleaning efficiency. However, Vaze [15] has expressed doubts about the efficiency of the road sweeper, because only some of the particles will be collected, and small particles will be released into the air. As the core of the dust suction system of a road sweeper, the dust collection system directly affects the dust collection efficiency of the sweeper and haze governance in cities. Chen et al. [16] improved the shape of the dust collection system, through a traditional prototype testing method, by placing wing panels parallel to the ground at the front and back of the sweeper. 
The duration of airflow action on the ground particles increased, owing to this placement.

Trial production prototype can test the manufacturing feasibility of product design. But it costs money and time. With the development of computer technology, computational fluid dynamics (CFD) technology has been employed, as a design guide, in the field of sanitation equipment. Xu et al. $[17,18]$ applied CFD method for the first-time ever to the design of a road sweeper. The authors proposed that the dust suction power can be improved by changing the shape of the horizontal section comprising the dust collection system. Using a parameter design method, Yang et al. [19] assessed the width, diameter, and inclination angle of the dust collection system, and determined the optimal combination of structural parameters. Structural design needs theoretical guidance. From the perspective of fluid mechanics, Zeng [20] analyzed (via simulations) and performed structural development of the flow field associated with the dust collection system of a highway sweeper. The results revealed that the optimized structure will prevent gas escape and, in turn, secondary pollution. Zhang et al. [21] found that the structural parameters of the external expansion area affect the accuracy of simulation computations, and the rational selection of parameters for the expansion area is particularly important for the calculation result and calculation accuracy.

The aforementioned studies are all based on the analysis of single gas-phase flow, thereby providing theoretical support for the structural design and simulation computation of a dust collection system. However, the presence of a solid phase under real conditions is ignored. Using a gas-solid two-phase liquid computing method, $\mathrm{Wu}$ [22] determined the running speed and system pressure drop of a dust collection system, and proposed an optimum operation-condition range. Changing the operational conditions is a good way to enhance the solid particle separation performance, because there is no structural interference. But the performance gains are limited. Wu [26] combined the computing method with the motion trail of particles, and optimized the angle and pressure drop of the back baffle. The results revealed that the optimal dust collection efficiency is achieved at an angle and pressure drop of $135^{\circ}$ and $2400 \mathrm{~Pa}$, respectively.

The principle of operation determines the structural characteristics. The above investigation and research are concentrated upon the effect of traditional vacuum cleaning system. The traditional vacuum type only utilizes the negative pressure delivery theory. We have proposed a novel structure which utilizes both negative and positive pressure mixed-delivery theory [23], and we call this novel structure the vacuum-blowing cleaning system. The particle behavior is more complicated in the mixedpressure field. To enhance the solid particle separation performance and to evaluate the motion characteristics of inhaled particles accurately, the interplay between geometrical parameters is the key to demonstrate the influence and mechanism clearly $[24,25]$. Therefore, the interplay between structural parameters should not be neglected for the dust collection efficiency of prediction and optimization.
In this work, the structural parameters required for the modeling of a vacuum-blowing cleaning system are investigated from the viewpoint of their influence on the dust collection efficiency. Specifically, the motion characteristics of inhaled particulates are evaluated using a computing method where a gas-solid two-phase liquid is considered. The total dust collection efficiency, grade dust collection efficiency, and average retention time are subsequently computed. Moreover, the optimal structure is determined based on uniform experiments and regression analysis considering the structural interplay, and the rationality of the numerical simulation is verified via experiments.

\section{Physical model and computation domain}

Physical model of the sweeper vehicle is shown in Figure 1a. For successful inhalation of ground particles during operation, a certain ground clearance (generally $10-15 \mathrm{~mm}$ ) must be maintained between the vacuum-blowing cleaning system and the ground. For the simulation performed in this work, the ground clearance is set to $10 \mathrm{~mm}$. Figure $1 \mathrm{~b}$ shows the operational principle of the vacuum-blowing cleaning system. Suction-inlet 1 is connected to a centrifugal fan, where the negative pressure generated allows clearance-induced suction against the surrounding air. Moreover, the incoming air from Blowing-inlet 4 blows the particulate matter toward Suction-inlet 1. Under the action of the applied blowing force, the negative pressure generated by Suction-inlet can suck the particles up easily with lower pressure. In our previous published papers, we have clarified the mechanism of the reverse blowing flow rate that acted on the Blowing-inlet, and we also calculated the optimized results [23]. So the Blowing-inlet helps enhance the cleaning efficiency and reduces the consumption of energy for centrifugal fan. Finally, the flowing air carries particles into the cleaning trunk, thereby resulting in particle elimination.

The air-inlet gap area of the vacuum-blowing cleaning system is small, and measurement and calculation of the air inflow velocity, incoming air flow, and pressure in the surrounding gap are difficult. Moreover, the gap is an interface between the inner flow field and outer space, where the air flow is complex. The difficulty in setting entrance boundary conditions can be overcome by designating the gap surrounding the dust collection system as an expansion area $[17-22,26]$ (see Fig. 2), and the structural parameters of expansion area are designed based on Reference [21]. Specifically, to simulate actual conditions, the four entrances of the expansion area are set to standard atmosphere pressure. The main structural parameters of the flow channel are shown in Table 1.

\section{Meshing and numerical methodology}

\subsection{Algorithm selection and model parameter setting}

To improve the computational accuracy, the finite volume method was employed for equation discretization, and a standard $k-\varepsilon$ turbulence model was applied [17-21,26]. 


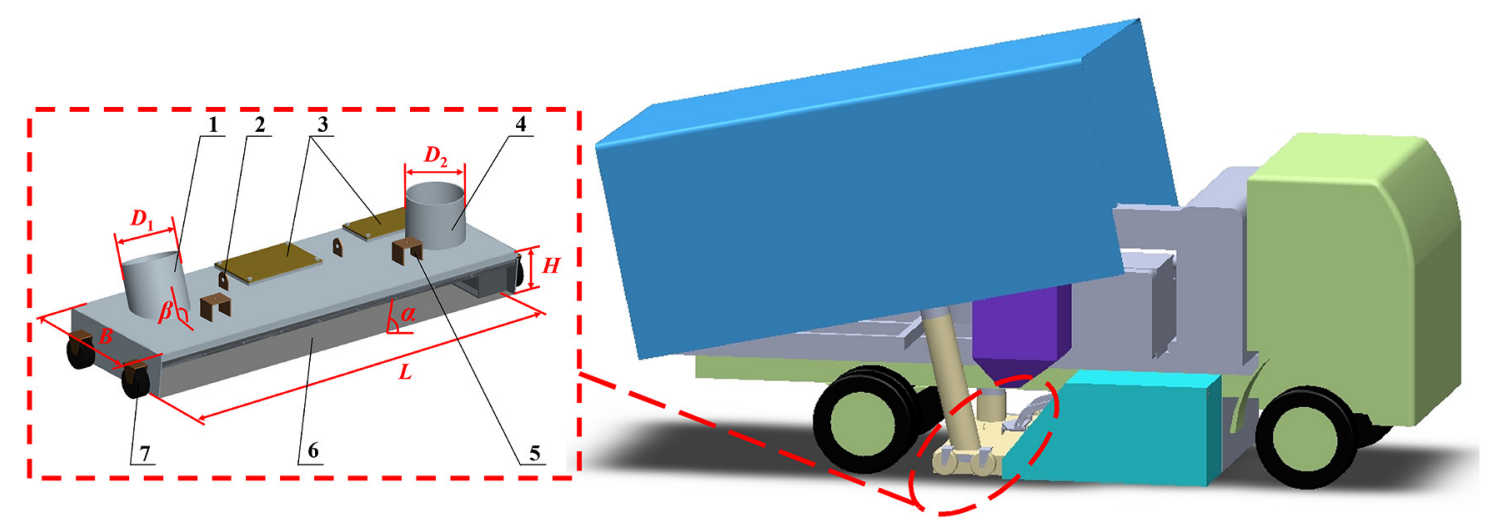

1-Suction-inlet; 2-Lifting lug; 3-Dust removal cover plate;

4-Blowing-inlet; 5-Base plate 6-Front baffle; 7-Return roller

(a)

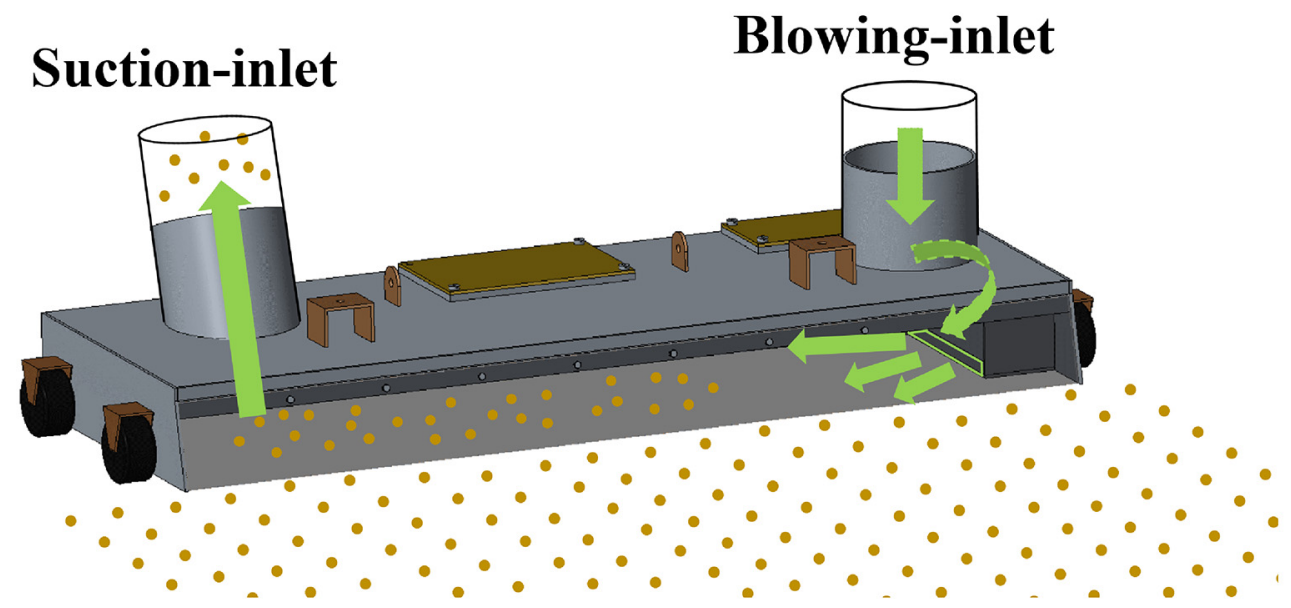

(b)

Fig. 1. Physical model. (a) Sweeper vehicle. (b) Vacuum-blowing cleaning system.

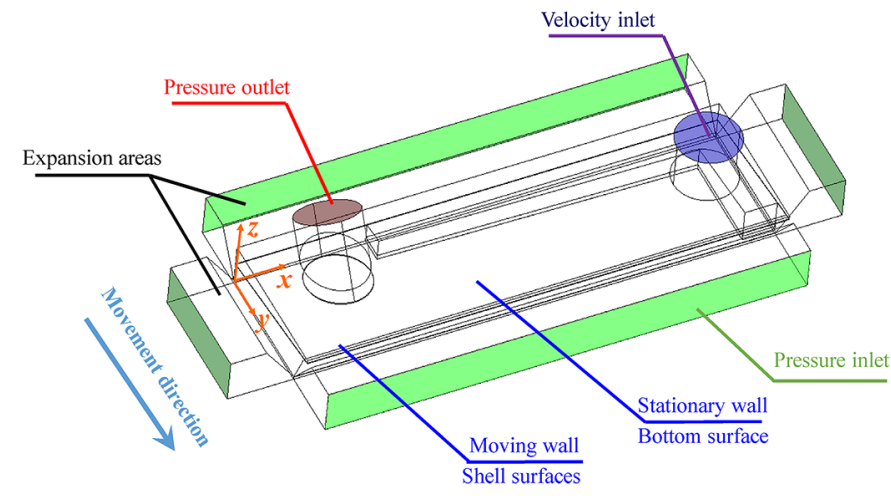

Fig. 2. CFD computation domain of the vacuum-blowing cleaning system.

The turbulence model is used to solve the flow in the core of turbulence. But to the near-wall treatment, the wall function is a semi-empirical description, and it is also a complement to the turbulence model. For our research, scalable wall functions was adopted [27]. Furthermore, an incompressible steady-state solution method was adopted for the internal part, and a second-order upwind difference scheme was chosen to discretize the governing equations. The pressure-velocity coupling and SIMPLE solution algorithm were selected in order to predict incompressible flow [28]. Boundary conditions were set as follows: for the flow field calculation, the suction-inlet vent, blowing-inlet, expansion area, and other boundary conditions were set as the pressure outlet, velocity inlet, pressure inlet, and wall surface, respectively. The average value of the pressure outlet was $-2300 \mathrm{~Pa}$ according to the measurement at the suction-inlet. The average value of the velocity inlet was $22.9 \mathrm{~m} / \mathrm{s}$, because the reverse blowing flow rate and the blowing-inlet diameter were $1871 \mathrm{~m}^{3} / \mathrm{h}$ and $170 \mathrm{~mm}$, respectively. The expansion areas connected the atmosphere, so the value of the pressure inlet was 0 . The wall surface at the bottom of the dust collection system was nonslipping stationary wall. The other wall surfaces were moving wall, and the value of them was $5 \mathrm{~km} / \mathrm{h}$, which simulated the driving status. 
Table 1. Structural parameters.

\begin{tabular}{lllllll}
\hline $\begin{array}{l}\text { Length } \\
L\end{array}$ & $\begin{array}{l}\text { Width } \\
B\end{array}$ & $\begin{array}{l}\text { Thickness } \\
H\end{array}$ & $\begin{array}{l}\text { Front baffle } \\
\text { angle } \\
\alpha\end{array}$ & $\begin{array}{l}\text { Suction-inlet } \\
\text { inclination angle } \\
\beta\end{array}$ & $\begin{array}{l}\text { Suction-inlet } \\
\text { diameter } \\
D_{1}\end{array}$ & $\begin{array}{l}\text { Blowing-inlet } \\
\text { diameter } \\
D_{2}\end{array}$ \\
\hline$(\mathrm{mm})$ & $(\mathrm{mm})$ & $(\mathrm{mm})$ & $\left(^{\circ}\right)$ & $\left(^{\circ}\right)$ & $(\mathrm{mm})$ & $(\mathrm{mm})$ \\
1400 & 450 & 130 & 60 & 120 & 170 & 170 \\
\hline
\end{tabular}

Table 2. Parameter setting for simulation calculation.

\begin{tabular}{llll}
\hline Name & Fluid phase & Name & Particle phase \\
\hline Fluid type & Air & Mass velocity $(\mathrm{kg} / \mathrm{s})$ & 0.5 \\
Compressibility & Incompressible & Particle size distribution & Rosin-Rammler \\
Grid quantity & 162874 & Min particle diameter $(\mu \mathrm{m})$ & 45 \\
Time response & Steady state & Max particle diameter $(\mu \mathrm{m})$ & 152 \\
Turbulence model & $k-\varepsilon$ & Median particle diameter $(\mu \mathrm{m})$ & 81 \\
Inflow boundary conditions & Pressure inlet Velocity inlet & Packing parameter & 5.95 \\
Open boundary conditions & Pressure outlet & Recovery coefficient & Normal value 0.95 \\
& & & Tangential value 0.85 \\
\hline
\end{tabular}

The discrete phase model (DPM) was used for describing the particle characteristics [26,29]. For turbulent fluctuations in the flow, the turbulent dispersion with stochastic tracking, discrete random walk model, and steady particle tracking were utilized, respectively. For particle treatment, the particles in this study were treated as spherical. The simulation calculation of the dust collection efficiency under actual working conditions, such as the diameter, density, etc., was performed in combination with the particle properties test results [30]. The particles were static and uniform spatial distribution on the floor $[22,26,29]$. For injection definition, the injection surface of particles was defined at the bottom surface of the front expansion area considering the sanitation device driving forward. Sand was the main particulate matter on the pavement [22]. Therefore, the particle properties of sand were adopted for simulation [31]. The DEM collision model was used to simulate the friction of particles through UDF (User Define Function). For particle-wall interaction, the wall boundary conditions were set as the "reflect" condition and the collision was assumed inelastic. The particles could be exported at the Suction-inlet 1 (Fig. 1), so this surface was set as the "escape" condition.

ANSYS Fluent 14.5 was selected as a solver for the flow field calculation. Table 2 is list of details for the parameter settings that applied to the simulation calculation.

\subsection{Grid discretization and fundamental assumption}

The structure of the vacuum-blowing cleaning system is complex and, hence, a structured grid partition is employed after the entire structure is divided into several sub-areas. Meanwhile, a relatively dense grid is chosen around the wall surface, to seize the velocity gradient at the boundary

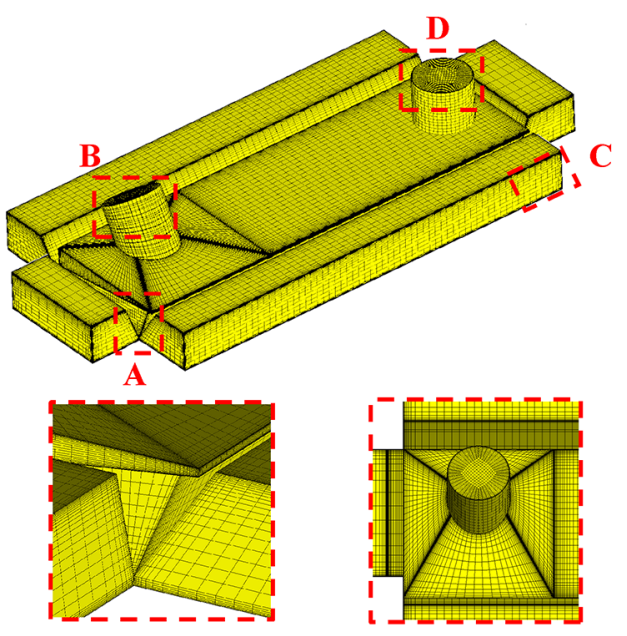

A
B
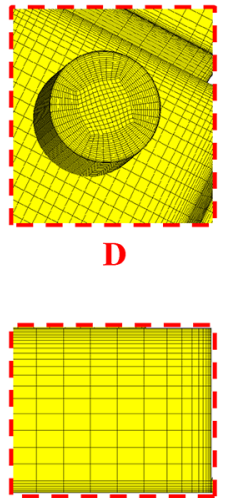

C
Fig. 3. Grid model and partial enlargement.

layer. The dense grid meets the exponential growth rule. The $y+$ is usually used for turbulence problems. The $y+$ is a non-dimensional distance to the wall, and it can be described as following:

$$
y+=\frac{u_{*} y}{v}
$$

Where $u *$ is the friction velocity, $y$ is the distance to the nearest wall, and $v$ is the local kinematic viscosity.

We find that the grid $y+$ of the boundary layer is 3 in our published paper [23]. It is suitable for the simulated calculation of vacuum-blowing cleaning system. Hence we adopt the grid of $y+=3$. The grid model is shown in Figure 3 . 


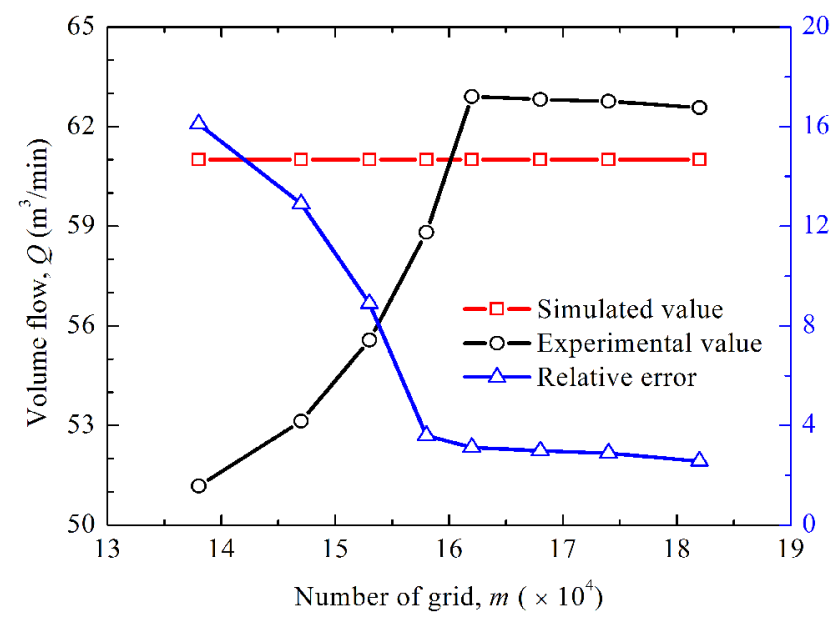

Fig. 4. Grid independence analysis.

The grid was divided by ANSYS ICEM 14.5. Figure 4 shows the independence analysis results of the structured grid. According to the figure, the simulation flow value at the suction-inlet vent increases gradually with increasing number of grid points, whereas the relative error decreases gradually. The simulation flow value at the vent and relative error converge (in general) for grid numbers over 160,000. The model considers ideal conditions for the simulation analysis, and neglects the flow loss resulting from the air leak. Therefore, the simulation analysis value is slightly larger than the actual experimental value. The number of grid points is set to 162,874 , based on the computation structure accuracy and computer processing power. This number of points yields (in general) a stable flow at the suction-inlet vent, and a small relative error (i.e., $3.12 \%$ ) between the simulation data and the experimental results.

To simplify the working process of dust collection during mobile operation of the vacuum-blowing cleaning system, the simulation model is simplified as follows: (i) The cleaning operation occurs without heat exchange between the air in the vacuum-blowing cleaning system and external air; (ii) The airflow in the dust collection system is incompressible and undergoes stable-state flow, and boundary conditions remain unchanged with the time; (iii) The expansion area is designated as the region surrounding the vacuum-blowing cleaning system, and the inlet pressure is set to standard atmospheric pressure; (iv) The vacuum-blowing cleaning system moves at a constant speed during operation, and dust particles are static unless being inhaled via the front inlet surface.

\section{Analysis of structure influence parameters}

\subsection{Suction-inlet diameter}

Considering the dimensional impact of the vacuumblowing cleaning system in the assembly of a road sweeper, the length, width, and height of the dust collection system have been changed only slightly. Of the four inlet surfaces comprising the dust collection system, the front inlet surface has the greatest impact on and is positively

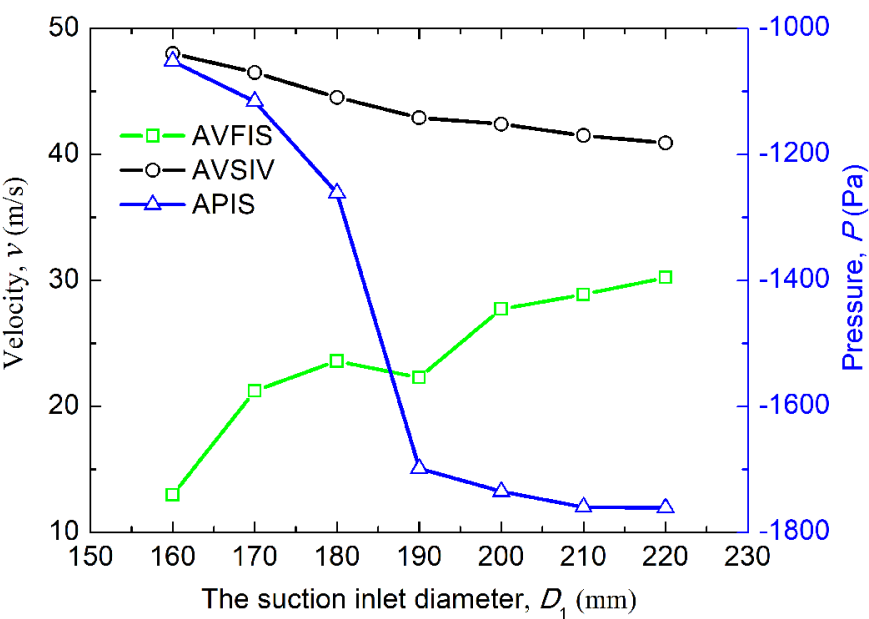

Fig. 5. Impact of the suction-inlet diameter on the velocity and pressure.

correlated with the dust collection efficiency [22]. The energy loss and dust collection performance are assessed by calculating the average velocity of the front inlet surface (AVFIS), average velocity of the suction-inlet vent (AVSIV), and average pressure of the inlet surface (APIS) $[19,32]$. Suppose $\alpha$ is $70^{\circ}$ and $\beta$ is $120^{\circ}$, respectively. The impact of the suction-inlet diameter on the average velocity of the front inlet surface, average velocity of the suctioninlet vent, and average pressure of the suction-inlet entrance is shown in Figure 5.

The average velocity of the front inlet surface increases continuously with increasing suction-inlet diameter. In addition, the average velocity of the suction-inlet vent and the average pressure of the suction-inlet entrance decrease with increasing diameter. This results from the fact that the pressure at the inlet vent is constant, entrance area increases with increasing diameter, and dust collection power increases continuously. Therefore, the average velocity of the front inlet surface and entrance increases, but the incremental flow value at the vent is less than the increase amplitude of the cross-sectional area; thus, the average velocity of the vent decreases. The increase in the suction-inlet diameter leads to a reduction in both the distance between the inlet and the front baffle and the linear loss of the inlet. The entrance is significantly impacted by the pressure of the vent and, hence, the pressure of the entrance gradually approaches the pressure of the vent. Consequently, the average pressure of the suction-inlet entrance decreases.

The total dust collection efficiency is the ratio of the quality of collected dust particles to the particles that enters the device. While the grade dust collection efficiency is the dust collection efficiency for different particle diameter. The number of particles can be monitored by software post-processing at different sections in the vacuum-blowing cleaning system. As the device goes forward, so the particles come into the device through the front ground. The bottom surface of the front expansion area, as an injection surface, injects the particles. The particles can escape through the suction-inlet surface. 


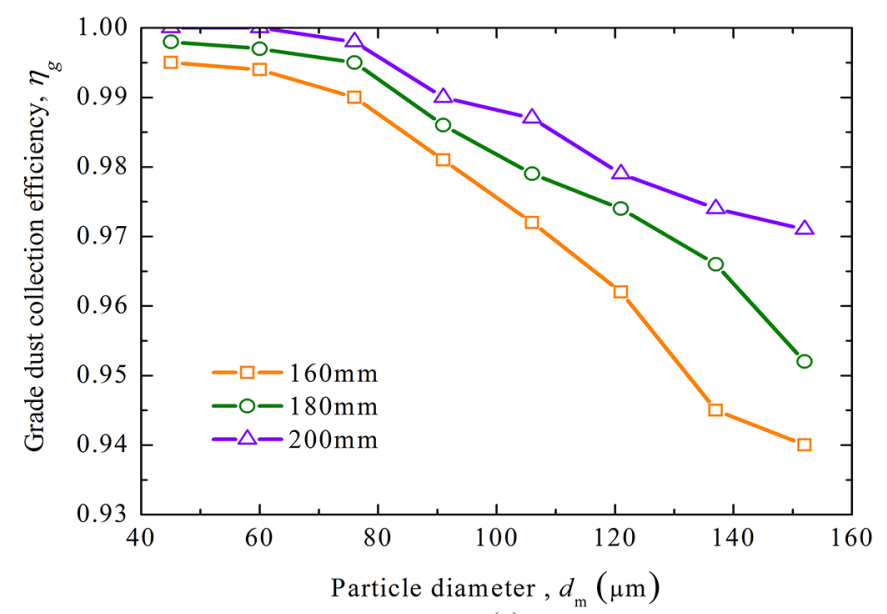

(a)

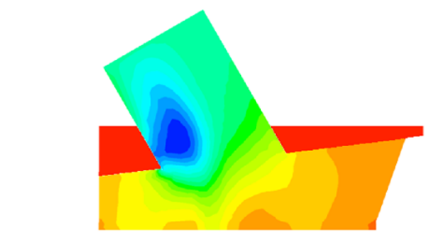

$D_{1}=160 \mathrm{~mm}$

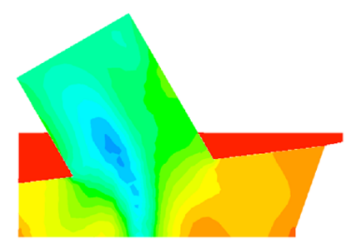

$D_{1}=180 \mathrm{~mm}$

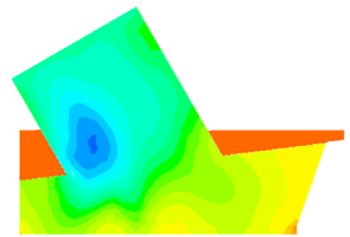

$D_{1}=200 \mathrm{~mm}$

Pressure

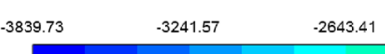

$-2045.24$

$-1447.08$

$-848.92$

$-250.75$

(b)

Fig. 6. Grade dust collection efficiency and pressure distribution for different suction-inlet diameters. (a) Grade dust collection efficiency. (b) Pressure distribution at the plane of $\mathrm{x}=500 \mathrm{~mm}$.

Through this method, we can calculated the number and get the total and grade dust collection efficiency [33].

The grade dust collection efficiency is an important indicator for assessing the vacuum-blowing cleaning system performance associated with the collection of different-sized particles. The grade dust collection efficiency and pressure distribution for different suction-inlet diameters are shown in Figure 6. As the figure shows, for a given inlet diameter, the efficiency decreases with increasing particle size. The largest reduction in the efficiency (i.e., from $99.7 \%$ to $93.9 \%$ ) occurs at an inlet diameter of $160 \mathrm{~mm}$. For a given particle size, the efficiency increases with increasing inlet diameter, with the most significant increase (i.e., $3.2 \%$ ) occurring at a particle size of $152 \mu \mathrm{m}$. These results indicate that changes in the suction-inlet diameter have a significant influence on the grade dust collection efficiency of the dust collection system. In the case of the inlet with a constant negative pressure, the inlet diameter is positively correlated with the dust collection power, which enhances the collection of particulate matter. The flow mobility increases with decreasing particle size. Compared with the mobility, the grade dust collection efficiency is less affected by changes in the diameter.

The average particle-retention time is defined as how long does the particle come in and out of the device. What's more, the average particle-retention time is employed to evaluate the particle dynamic characteristics, and it can be obtained by monitoring of particle trajectories [34]. However, different structure has different retention time.
There has no critical value of the retention time after which particles cannot be collected [22,23]. In order to realize the synchronous transportation of different-sized particles in the industrial process, it is necessary to match the mean residence time of different-sized particles with the transportation completion time $[35,36]$. Therefore, the average particle-retention time represents another important indicator for assessing whether particles in the dust collection system can be smoothly delivered to garbage. The retention time of particle is more uniform is better, and the shortest average retention time possible is desired. In view of this, different model requires the calculation of the average particle-retention time [37].

Figure 7 shows the average retention time of differentsized particles associated with three suction-inlet diameters (i.e., 160, 180, and $200 \mathrm{~mm}$ ). For the same suction-inlet diameter, the average retention time increases with increasing particle diameter. However, for the same particle diameter, it decreases with increasing inlet diameter. This indicates that, for different grain sizes, the running time of particles in the dust collection system varies. The average retention time of small particles is lower than that of large particles. Therefore, small particles are more easily collected than large particles. The average retention time associated with the $200-\mathrm{mm}$ inlet changes more slowly than the time associated with the $160-\mathrm{mm}$ inlet. The increase in the suction-inlet diameter yields significant improvement in a certain phase of the particlecollection performance. As previously shown in Figure 7, 


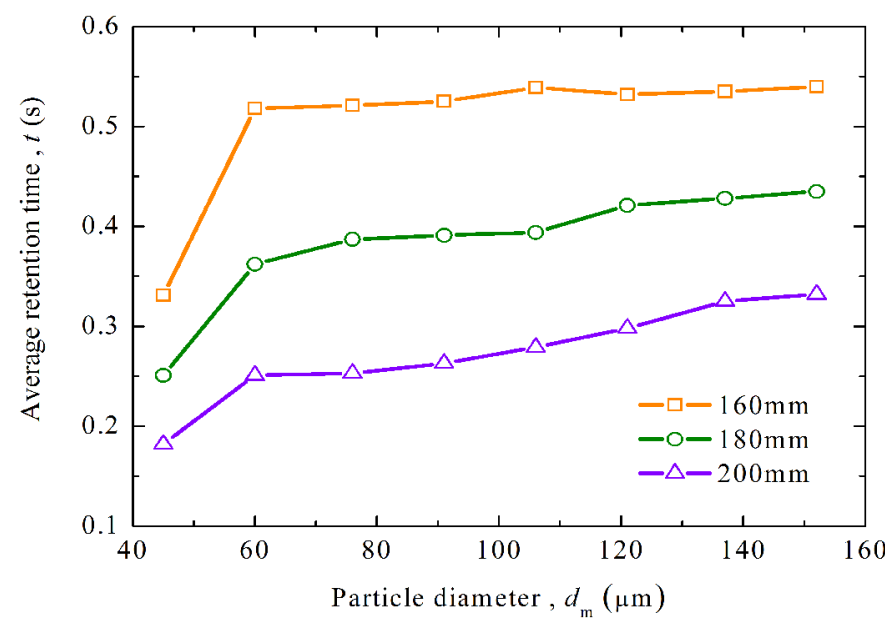

Fig. 7. Average retention time of different-sized particles at the suction-inlet.

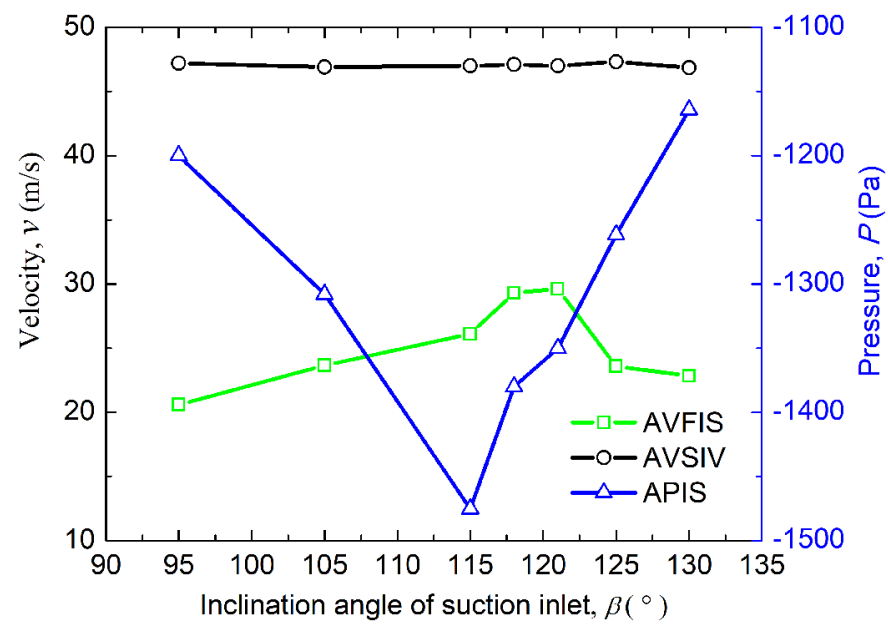

Fig. 8. Dependence of the velocity and pressure on the inclination angle $\beta$ of the suction-inlet.

an infinite increase in the suction-inlet diameter yields negligible contribution to the dust collection efficiency and the positive diameter-efficiency correlation is destroyed. Thus, a dynamic equilibrium period is realized and, hence, increasing the inlet diameter only yields no improvement in the dust collection efficiency.

\subsection{Inclination angle of suction-inlet}

Suppose D1 is $190 \mathrm{~mm}$ and $\alpha$ is $70^{\circ}$, respectively. The dependence of the velocity and pressure on the inclination angle $\beta$ of the suction-inlet is shown in Figure 8 . As the figure shows, when $\beta$ increases, the average velocity of the front inlet surface increases initially and then decreases, whereas the pressure of the suction-inlet decreases initially and then increases. However, the average velocity of the inlet vent changes only slightly. These results from the fact that, for $\beta$ smaller than $105^{\circ}$, the internal air flows smoothly, energy loss is small, dust collection power improves, average velocity of the front inlet surface increases, and the static pressure of the entrance decreases. For $\beta$ larger than $105^{\circ}$, the local resistance in the inner chamber increases, thereby resulting in increased energy loss and, hence, a decrease in the average velocity of the front inlet surface. However, the sectional area of the entrance increases with increasing $\beta$. The entrance is only slightly affected by the pressure of the vent and, therefore, the pressure at the entrance of the suction-inlet increases. For inclination angles ranging from $90^{\circ}$ to $130^{\circ}$, the velocity of the suction-inlet vent remains constant, owing to the: constant vent pressure, and the approximately equal resistances resulting from the increase in the sectional-area entrance, increase in the dust collection power, and roughness of the airflow.

The grade dust collection efficiency and pressure distribution for different inclination angles $\left(90^{\circ}, 115^{\circ}\right.$, and $130^{\circ}$ ) of the suction-inlet are shown in Figure 9. As the figure shows, for a fixed particle size and increasing inclination angle, the efficiency increases initially and then decreases. The largest decrease in efficiency (i.e., 2.3\%) occurs at a particle size of $152 \mu \mathrm{m}$ and an inclination angle of $90^{\circ}$. For the same inclination angle, the efficiency decreases with increasing particle diameter, especially for an angle of $90^{\circ}$, where the largest drop (from $99.7 \%$ to $94.1 \%$ ) occurs. The change in the inclination angle of the suction-inlet has a significant impact on the dust collection efficiency, with excessively large or small angles yielding low efficiency. A suitable inclination angle contributes to the smoothness of air flow, energy loss reduction, and realization of optimal dust collection efficiency.

The average retention time of particles determined for three different inclination angles $\left(90^{\circ}, 115^{\circ}\right.$, and $\left.130^{\circ}\right)$ of the suction-inlet is shown in Figure 10. For a given inclination angle, the average retention time increases with increasing particle diameter. For particles of different sizes, the running time of particles inside the dust collection system varies, and the retention time is positively correlated with the particle size. Values of $0.1641,0.1660$ and $0.1711 \mathrm{~s}$ are obtained for $\Delta t$ (i.e., the difference in the average retention time) corresponding to inclination angles of $90^{\circ}, 115^{\circ}$ and $130^{\circ}$, respectively. Therefore, the increase in the inclination angle of the suction-inlet has only a slight impact on the average particle-retention time. Furthermore, compared with the variation in particle diameter, the increase in the suction-inlet inclination angle exerts less influence on the retention time.

\subsection{Inclination angle of front baffle}

Suppose D1 is $190 \mathrm{~mm}$ and $\beta$ is $115^{\circ}$, respectively. The dependence of the velocity and pressure on the inclination angle $\alpha$ of the front baffle is shown in Figure 11. As the figure shows, when $\alpha$ increases, the average velocity of the front inlet surface increases initially and then decreases, whereas the opposite trend is observed for the average pressure. However, the average velocity of the inlet changes only slightly. This results from the fact that, for inclination angles smaller than $100^{\circ}$, when the angle increases, 


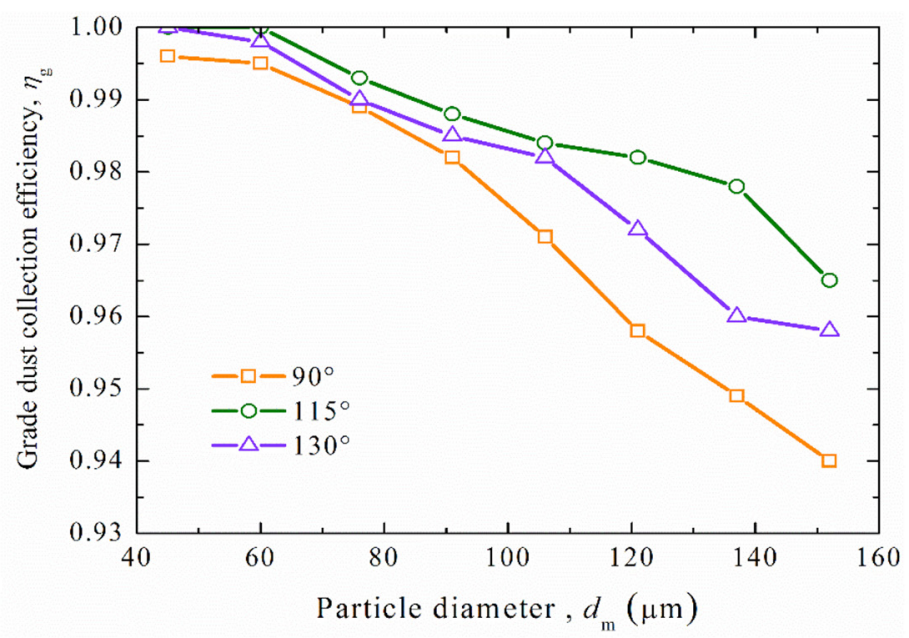

(a)

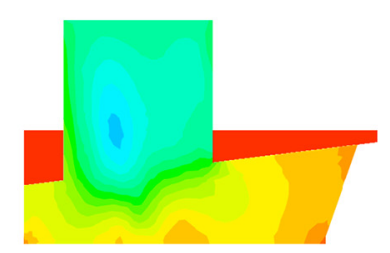

$\beta=90^{\circ}$

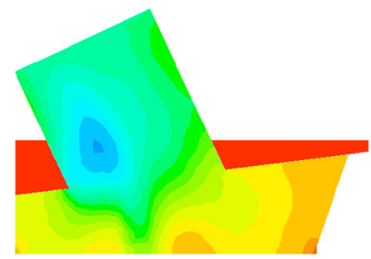

$\beta=115^{\circ}$

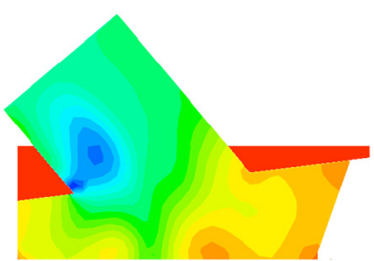

$\beta=130^{\circ}$

Pressure

[ pascal]

$-3987.07 \quad-3380.02$

$-2772.98$

$-2165.93$

$-1558.89$

$-951.84$

$-344.80$

(b)

Fig. 9. Grade dust collection efficiency and pressure distribution for different inclination angles of the suction-inlet diameters. (a) Grade dust collection efficiency. Pressure distribution at the plane of $\mathrm{x}=500 \mathrm{~mm}$.

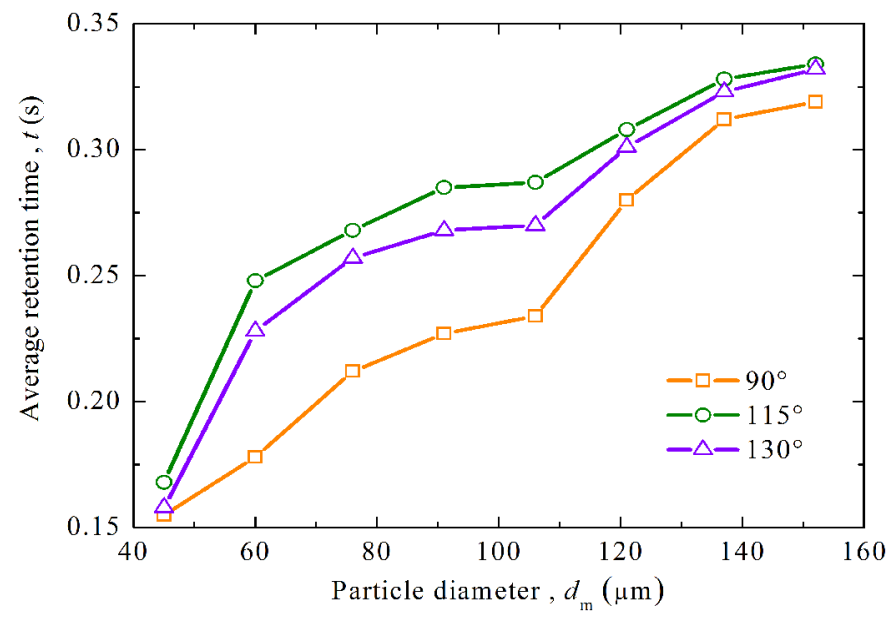

Fig. 10. Average retention time of particles determined for different inclination angles of the suction-inlet.

the internal air flows smoothly, energy loss is small, dust collection power improves, average velocity of the front inlet surface increases, and static pressure of the entrance decreases. If the inclination angle $\alpha$ is larger than $100^{\circ}$, the increase in the local resistance (owing to the reduction in the volume of the inner chamber) has significant impact on

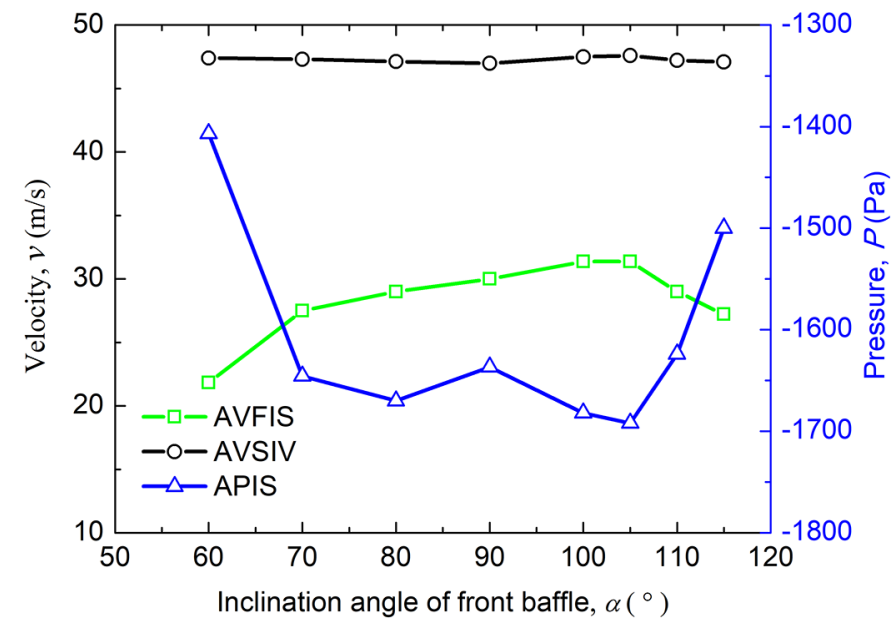

Fig. 11. Dependence of the velocity and pressure on the inclination angle $\alpha$ of the front baffle.

the flow field. This results in a velocity drop of the front inlet surface. The external impact on the inner pressure increases significantly, and the average pressure of the suction-inlet entrance increases. The velocity of the inlet vent remains almost unchanged for $\alpha$ values ranging from $50^{\circ}$ to $120^{\circ}$, owing to an increase in the local resistance in 


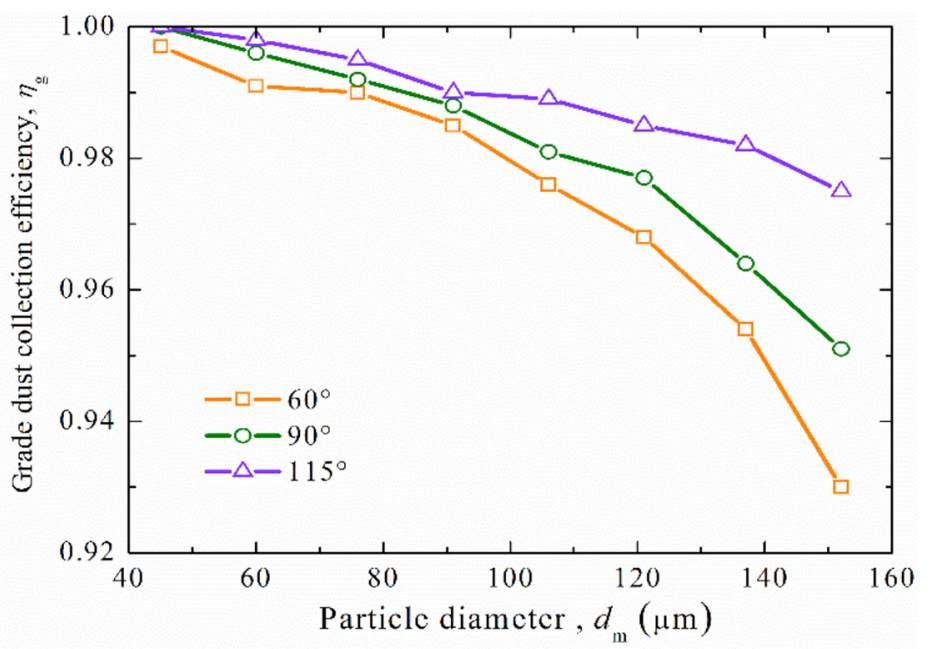

(a)

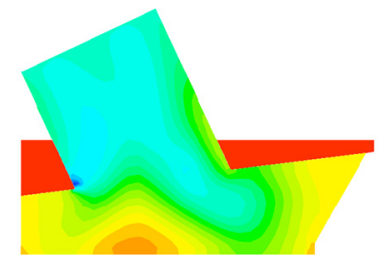

$\alpha=60^{\circ}$

Pressure

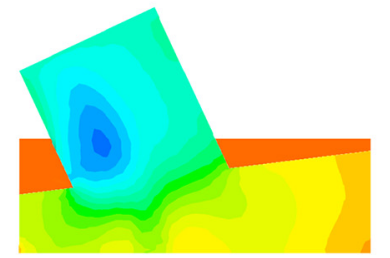

$\alpha=90^{\circ}$

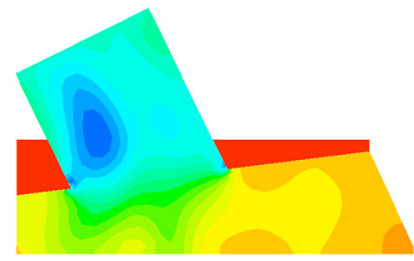

$\alpha=115^{\circ}$

[ pascal ]

(b)

Fig. 12. Grade dust collection efficiency and pressure distribution for different inclination angles of the front baffle. (a) Grade dust collection efficiency. (b) Pressure distribution at the plane of $x=500 \mathrm{~mm}$.

the inner chamber. Consequently, the energy loss increases with decreasing volume of the chamber, but the smoothness of the air flow offsets the energy loss resulting from the small space.

The grade dust collection efficiency and pressure distribution for different inclination angles $\left(60^{\circ}, 90^{\circ}\right.$, and $115^{\circ}$ ) of the front baffle are shown in Figure 12. The dust collection efficiency calculated for different inclination angles of the front baffle are shown in Figure 12. As the figure shows, for a given particle diameter, the efficiency decreases when the inclination angle decreases from $115^{\circ}$ to $60^{\circ}$ (for example, a particle size of $152 \mu \mathrm{m}$ corresponds to a $4.5 \%$ decrease). Similarly, for the same inclination angle, the efficiency decreases with increasing particle diameter. The most significant drop (from $99.7 \%$ to $93.0 \%$ ) occurs for diameters ranging from 45 to $152 \mu \mathrm{m}$, and a given inclination angle of $60^{\circ}$. This results from the fact that, similar to the suction-inlet inclination angle, a suitable baffle inclination angle contributes to the smoothness of air flow and improvement of the dust collection efficiency.

The average retention time of the particles is determined for three different inclination angles (i.e., $60^{\circ}, 90^{\circ}$, and $115^{\circ}$ ) of the front baffle (see Fig. 13). As the figure shows, for a given inclination angle, the average retention time increases with increasing size of the particles.

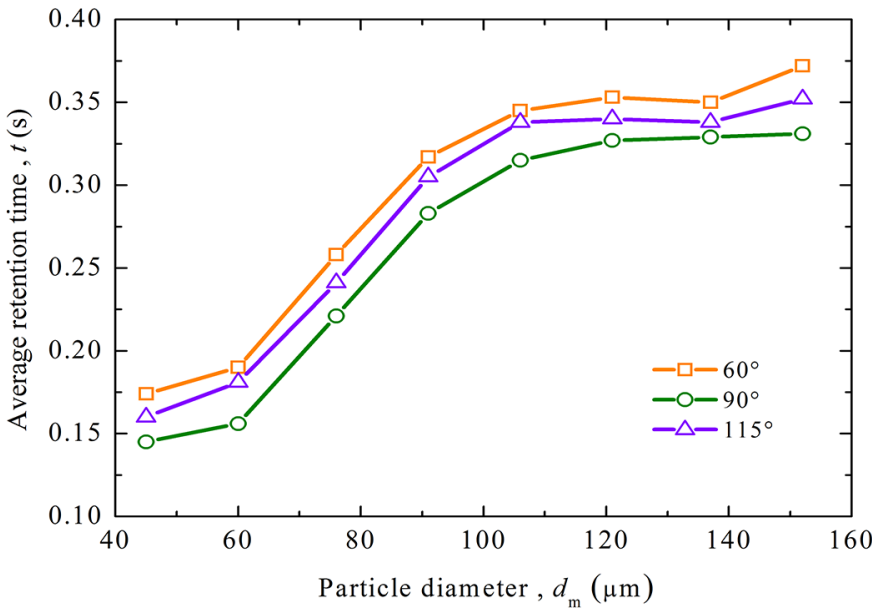

Fig. 13. Average retention time of particles corresponding to three different inclination angles of the front baffle.

This results from the fact that the top cover of the dust collection system must be connected to the vehicle body. The cover size remains unchanged, and the increase in the inclination angle of the baffle leads to an increase in the width of the vacuum-blowing cleaning system and the 
Table 3. Factor level table of structural parameters and experimental results.

\begin{tabular}{lllll}
\hline NO. & $\begin{array}{l}x_{1} \\
\text { Suction-inlet } \\
\text { diameter }(\mathrm{mm})\end{array}$ & $\begin{array}{l}x_{2} \\
\text { Inclination angle } \\
\text { of suction-inlet }\left(^{\circ}\right)\end{array}$ & $\begin{array}{l}x_{3} \\
\text { Inclination angle } \\
\text { of front baffle }\left(^{\circ}\right)\end{array}$ & $\begin{array}{l}\text { Average velocity of front } \\
\text { inlet surface }(\mathrm{m} / \mathrm{s})\end{array}$ \\
\hline 1 & 187 & 97 & 76 & 28.06 \\
2 & 187 & 123 & 110 & 28.81 \\
3 & 201 & 123 & 76 & 25.88 \\
4 & 201 & 84 & 110 & 29.21 \\
5 & 173 & 97 & 93 & 23.59 \\
6 & 159 & 110 & 59 & 27.71 \\
7 & 173 & 84 & 59 & 25.95 \\
8 & 201 & 97 & 93 & 31.09 \\
9 & 159 & 84 & 93 & 22.52 \\
10 & 187 & 110 & 76 & 31.58 \\
11 & 173 & 110 & 59 & 25.87 \\
12 & 159 & 123 & & 23.69 \\
\hline
\end{tabular}

volume of the chamber. Therefore, the average retention time of particles in the vacuum-blowing cleaning system increases slightly.

\section{Enhancement of particle collection efficiency considering the structural interplay}

\subsection{Experiment design and multiple regression model building}

The suction-inlet diameter, suction-inlet inclination angle, and front baffle inclination angle are evaluated. This evaluation is combined with uniform design and multiple regression analysis, where the criteria of a $\mathrm{U}_{12}\left(4^{3}\right)$ uniform design table are employed (see Tab. 3 for the calculation results) [38].

Considering the interaction among parameters and the quadratic component of the single factor, a ternary quadratic linear regression equation is adopted for modeling [38-40], as shown in equation (2).

$$
y=b_{0}+\sum_{i=1}^{n} b_{i} x_{i}+\sum_{i=1}^{n} b_{i i} x_{i}{ }^{2}+\sum_{i<j} b_{i j} x_{i} x_{j}
$$

Where, $n$ : number of factors, $y$ : predicted value, $x_{i}$ : influence factor, $b_{0}$ : constant, $b_{i i}$ : quadratic coefficient, and $b_{i j}$ : interaction term coefficient.

Equation (2) is formulated via stepwise regression of the quadratic polynomials. The resulting ternary quadratic regression equation describing the front inlet surface average velocity of the vacuum-blowing cleaning system relative to the diameter of the suction-inlet, inclination angle of the inlet, and inclination angle of the front baffle is:

$$
\begin{aligned}
y= & 1787.8240-19.8143 x_{1}+4.9474 x_{2}-5.7579 x_{3} \\
& +0.0492 x_{1}^{2}-0.0356 x_{2}^{2}-0.0135 x_{3}^{2} \\
& -0.0009 x_{1} x_{2}+0.0272 x_{1} x_{3}+0.0306 x_{2} x_{3}
\end{aligned}
$$

\subsection{Significance test of regression equation}

The multiple linear regression equation composed of structural parameters describing the vacuum-blowing cleaning system is formulated. This equation is then subjected to a significance test (in this case, an $F$ test) that considers the linear relation between the dependent variable y and the three independent variables [41]. Table 5 shows the variance analysis of the structural-parameter linear regression equation that describes the vacuumblowing cleaning system. Based on the table of $F$ values (i.e., Tab. 4), $F_{0.05}(9,2)=19.38<49.38$, which is indicative of a significant linear relation between the average velocity of the front inlet surface of the vacuum-blowing cleaning system and (i) suction-inlet diameter, (ii) inclination angle of the suction-inlet and (iii) inclination angle of the front baffle. In other words, the ternary quadratic regression is significant.

\subsection{Identifying interaction effects between the main structural parameters}

The significance of the regression equation is determined. Using the determined values, the significance of the linear relation between each factor $x$ and the dependent variable $y$ (see Eq. (2)) is ascertained via the $t$-test method [39] (see Tab. 5 for the corresponding results). The $t_{\mathrm{bi}}(i=1,2 \ldots 9)$ is partial regression coefficient.

Based on the table of $t$ values (see Tab. 6), $t_{0.05(2)}=4.303$. Valid results are obtained for $\left|t_{b_{6}}\right|<t_{0.05(2)}$ only, indicating that the partial regression coefficient $b_{7}$ has negligible impact on the regression equation and should therefore be eliminated. Considering the correlation among independent variables, insignificant independent variables should be eliminated in a stepwise manner $[40,42]$. The final regression equation describing the average velocity of the front inlet surface, diameter of the suction-inlet, inclination angle of the suction-inlet, and inclination angle of the front 
Table 4. Variance analysis of linear regression equation composed of structural parameters describing the vacuumblowing cleaning system.

\begin{tabular}{lcccc}
\hline Source of variance & Degree of freedom & Quadratic sum of deviation & Variance & $F$ value \\
\hline Regression & 9 & 93.37 & 10.37 & 49.38 \\
Partial regression & 2 & 0.41 & 0.21 & \\
Total & 11 & 93.78 & & \\
\hline
\end{tabular}

Table 5. $t$ Statistics.

\begin{tabular}{lllllllll}
\hline$t_{b_{1}}$ & $t_{b_{2}}$ & $t_{b_{3}}$ & $t_{b_{4}}$ & $t_{b_{5}}$ & $t_{b_{6}}$ & $t_{b_{7}}$ & $t_{b_{8}}$ & $t_{b_{9}}$ \\
\hline 11.257 & 10.117 & 8.705 & 11.765 & 7.342 & 4.756 & 0.272 & 10.343 & 10.823 \\
\hline
\end{tabular}

Table 6. Standardization of regression analysis coefficients.

\begin{tabular}{lrrrrrrrr}
\hline & \multicolumn{1}{c}{$x_{1}$} & \multicolumn{1}{c}{$x_{2}$} & \multicolumn{1}{c}{$x_{3}$} & \multicolumn{1}{c}{$x_{1}{ }^{2}$} & \multicolumn{1}{c}{$x_{2}{ }^{2}$} & \multicolumn{1}{c}{$x_{3}{ }^{2}$} & \multicolumn{1}{c}{$x_{1} x_{3}$} & $x_{2} x_{3}$ \\
\hline Regression coefficient & -19.9410 & 5.0444 & -5.8940 & 0.0492 & -0.0367 & -0.0128 & 0.0275 & 0.0303 \\
Standardized result & -111.6514 & 26.2263 & -40.0728 & 99.2577 & -39.6069 & -14.8047 & 36.2755 & 25.3123 \\
\hline
\end{tabular}

Table 7. Comparison of the grade dust collection efficiency $\eta_{\mathrm{g}}$ before and after optimization.

\begin{tabular}{lllllllll}
\hline Grain size $(\mu \mathrm{m})$ & 45 & 60 & 76 & 91 & 106 & 121 & 137 & 152 \\
\hline Optimized model & $99.03 \%$ & $98.75 \%$ & $99.13 \%$ & $98.75 \%$ & $99.08 \%$ & $95.02 \%$ & $94.15 \%$ & $93.73 \%$ \\
Original model & $99.00 \%$ & $98.65 \%$ & $97.21 \%$ & $96.05 \%$ & $95.84 \%$ & $93.62 \%$ & $92.35 \%$ & $90.21 \%$ \\
\hline
\end{tabular}

baffle is given as follows:

$$
\begin{aligned}
y= & 1799.9666-19.9410 x_{1}+5.0444 x_{2}-5.8940 x_{3} \\
& +0.0492 x_{1}^{2}-0.0367 x_{2}^{2}-0.0128 x_{3}^{2} \\
& +0.0275 x_{1} x_{3}+0.0303 x_{2} x_{3}
\end{aligned}
$$

\subsection{Structural-parameter optimization and numerical verification}

The parameter degree of influence is determined via standardization of the regression coefficients comprising equation (3) (see Tab. 6 for the standardized regression coefficients). With respect to the degree of influence on the test index (i.e., the average velocity of the front inlet surface), the order of the terms comprising the regression equation can be determined from the absolute values of the standardized regression coefficients. These coefficients can be written in descending order of this influence, that is, $x_{1}>x_{1}{ }^{2}>x_{3}>x_{2}{ }^{2}>x_{1} x_{3}>x_{2}>x_{2} x_{3}>x_{3}{ }^{2}$. The maximum of the regression equation is determined (in combination with MATLAB2014), and is realized for the following structural-parameter combination: suction-inlet diameter $x_{1}=201.0 \mathrm{~mm}$, suction-inlet inclination angle $x_{2}=110.7^{\circ}$, and front baffle inclination angle $x_{3}=106.2^{\circ}$.

A comparison of 12 test-result sets reveals poor matching of the parameters and, hence, the feasibility of the structural-parameter sets is determined via tests that consider these parameters. Considering actual engineering dimensions and machining precision, the following set of structural parameters is designated: inlet diameter $x_{1}=200.0 \mathrm{~mm}$, inclination angle of suction-inlet $x_{2}=110^{\circ}$, and inclination angle of front baffle $x_{3}=105^{\circ}$. These parameters are used in the structural-parameter matching for the vacuum-blowing cleaning system, and the calculation model is re-verified. The dust collection efficiency values determined before and after optimization are then compared (see Tab. 7).

The total dust collection efficiency can be determined from:

$$
\eta_{T}=\sum_{n=1}^{\infty}\left(\eta_{d} \bullet f_{d}\right)
$$

Where, $\eta_{\mathrm{T}^{-}}$total dust collection efficiency, $f_{\mathrm{d}}-$ proportion of mass fraction corresponding to different grain sizes, and $\eta_{\mathrm{d}}-$ grade dust collection efficiency. 


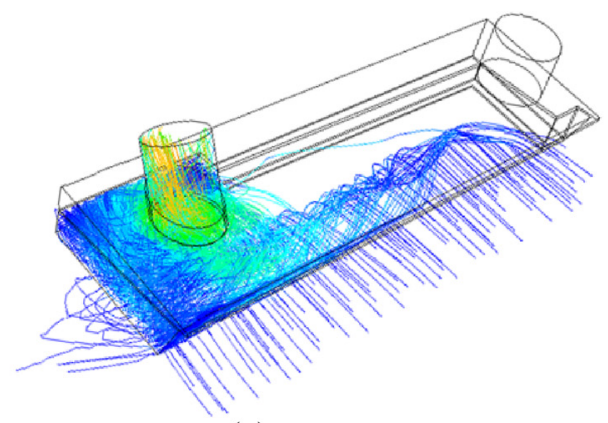

(a)

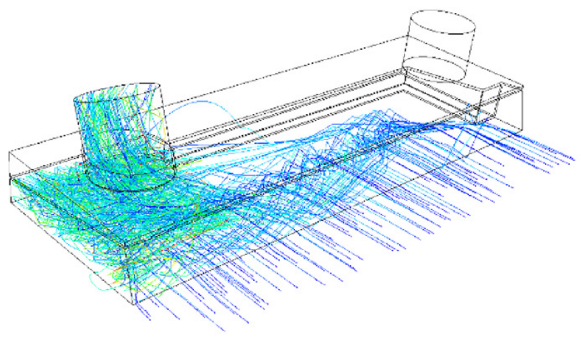

(b)

Particle Residence Time

0.00

0.18

0.36

0.55

0.73

0.91

[s]

Fig. 14. Motion trail and retention time of particles in the dust collection system before and after structure optimization. (a) Before optimization. (b) After optimization.

The total dust collection efficiency of the vacuumblowing cleaning system before and after optimization is $89.92 \%$ and $96.10 \%$ (i.e., $6.18 \%$ higher), respectively. Figure 14 shows the motion trail and retention time of particles in the vacuum-blowing cleaning system with the optimized structure. The trail of particles in the figure shows that (i) the leakage of dust in the right front of the driving direction is effectively controlled, and (ii) owing to reverse blow at the left of the vacuum-blowing cleaning system, all particles are blown to the region near the suction-inlet at the right and are inhaled under a negative pressure effect. At the same time, the optimized results show that retention time of particle is more uniform is better. In order to realize the synchronous transportation of different-sized particles in the industrial process, it is necessary to match the mean residence time of differentsized particles with the transportation completion time $[35,36]$. In this way, we can avoid the phenomena of particles back mixing and enhance the dust collection efficiency.

\section{Experimental verification}

The model machine of the vacuum-blowing cleaning system need rework because of structural improvements. Considering the impact on traffic and the potential safety hazard, a school is taken as the test site (see Fig. 15), which is designed as a road surface uniformly covered with dust particles. To avoid the complexity of testing that installed on the sweeper vehicle, the vacuum-blowing cleaning system is tested by adding centrifugal and axial flow fan. The centrifugal fan is used to provide negative pressure, which connects to the suction-inlet. The axial flow fan is used to provide positive pressure, which connects to the blowing-inlet and produces the reverse blowing flow rate. The type of the centrifugal fan is 9-26NO.3.3A (Liaoning Jinfeng Blower Co., Ltd, China). The value of the pressure can be easily controlled by frequency converter, and the regulated value is $-2300 \mathrm{~Pa}$. Similarly, the value of the reverse blowing flow rate is adjusted to $1871 \mathrm{~m}^{3} / \mathrm{h}$. The experiment is started, after the fans are stable.
The movement of the vacuum-blowing cleaning system is powered by a forklift.

The experimental verification is divided into two parts. One is a contrastive analysis of velocity, and the other is a contrastive analysis of dust collection efficiency. For velocity analysis, the two measure points are tested at the same time (see Fig. 15c). The location distribution of the first measure point $\mathrm{A}$ is at the $x=200 \mathrm{~mm}$ and $y=325 \mathrm{~mm}$. The second measure point $\mathrm{B}$ is at the $x=700 \mathrm{~mm}$ and $y=325 \mathrm{~mm}$. The two measuring points are all $20 \mathrm{~mm}$ higher than the ground surface. The KasdaKV621 hot-wire anemometers (Tianjin Kasda Instruments Co.,Ltd, China) were adopted for measuring speed, and they were fixed by the flange. The data acquisition instrument WS-5921/U60232, Beijing Wavespectrum Science \& Technology Co., Ltd, was also used. A computer with a Vib'SYS program was used together with the data acquisition instrument.

The road surface uniformly covered with dust particles, and the distribution density was $0.15 \mathrm{~kg} / \mathrm{m}^{2}$. We got the dust particles of the experiment from tertiary highway on the basis of the Reference [22]. Though the particles trajectory within the mechanical equipment were not be tracked, we could use velocity calibration and dust quality weighing method for experimental verification. The forklift moved at a speed of $6 \mathrm{~km} / \mathrm{h}$, and the contrastive analysis of experimental and simulation data is shown in Figure 16. The value of Measure point A is larger than Measure point B. The Measure point B is far from the suction-inlet, so it has low vacuum degree. The experimental data were a little lower than simulation one. The reasons are as follows. Firstly, the different sizes and properties adopt different dust particle models, which leads to different calculation results of the particle trajectories. The different particle trajectories also results in a statistics error for large quantity of particles in suction-inlet. Secondly, the simulation is carried out in an ideal environment, so it has not the leakage of air. In reality, the equipment can not be completely sealed and without leakage, there must be pressure loss. These factors are the main reasons, which leads to the simulation data is higher than experimental one. The comparison of 


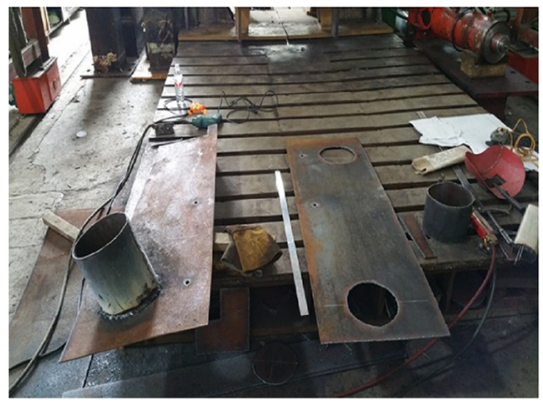

(a)

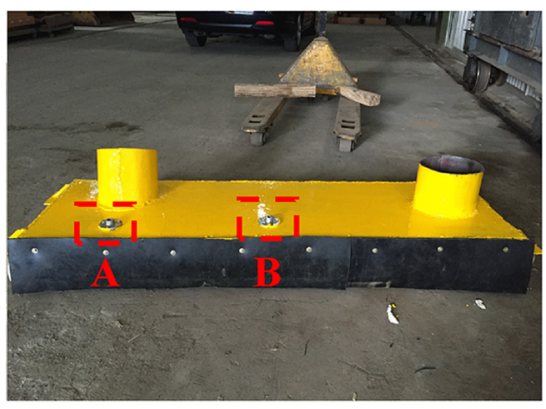

(b)

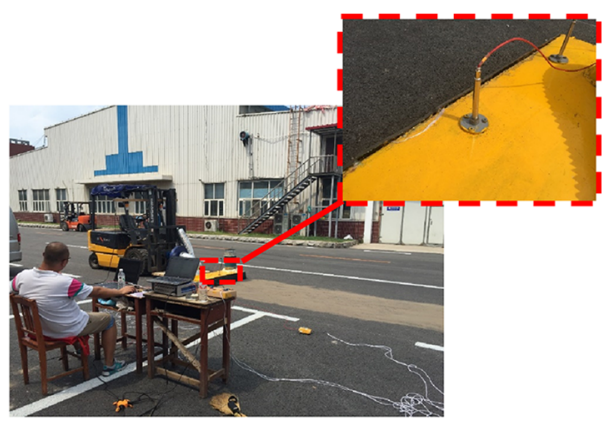

(c)

Fig. 15. Prototype and site test. (a) Trial-manufacture. (b) Model machine. (c) Site test.

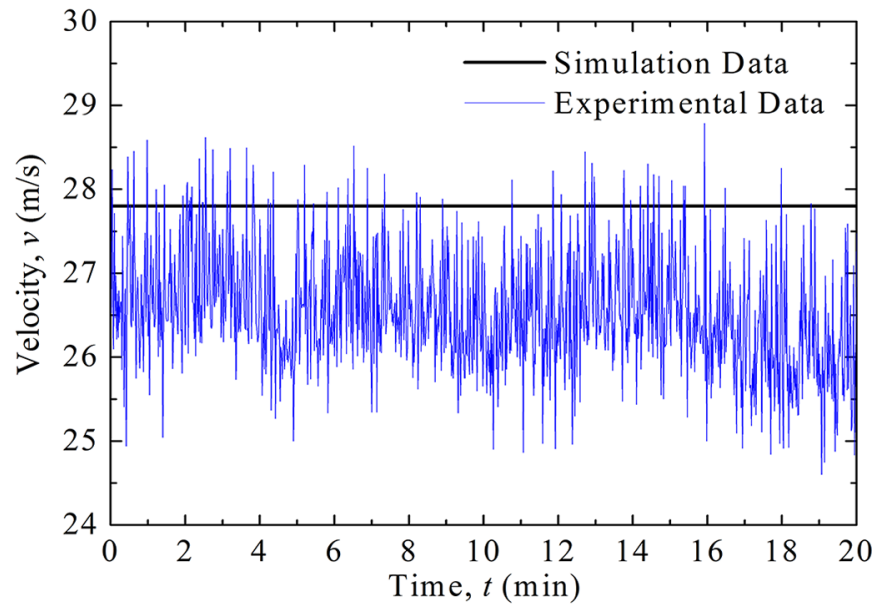

(a)

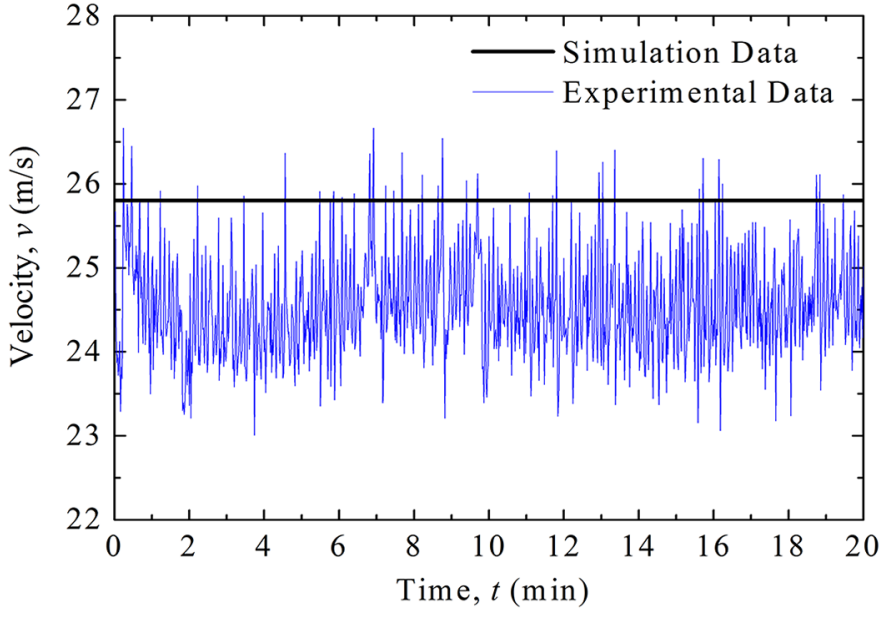

(b)

Fig. 16. Contrastive analysis of experimental and simulation data, $v_{\mathrm{t}}=6 \mathrm{~km} / \mathrm{h}$. (a) Measure point A. (b) Measure point B.

experimental and simulation data at the velocity of $6 \mathrm{~km} / \mathrm{h}$ preliminarily indicates that the CFD simulation method is authentic.

For dust collection efficiency analysis, using the method of mass calculation, the dust collection efficiency of the vacuum-blowing cleaning system is defined as the ratio of the quality of ground particles before and after dust collection $[22,26]$. Although the process of collecting and weighing particles is inevitably affected by human error, the obtained results are significant. The dust collection efficiency measurement results and CFD simulation results are compared in Figure 17.

In each case, the dust collection efficiency decreases with increasing speed of the vehicle. The increase in the running speed of the vacuum-blowing cleaning system leads to an increase in the speed of the vacuum-blowing cleaning system relative to that of the particles. This is detrimental to dust collection and leads to a decrease in the dust collection efficiency. The relative error associated with the efficiency is determined from equation (6). The maximum allowable dust collection performance error of the dust collection system is $10 \%[22,26,29,43]$. This requirement is satisfied by values of $12.31 \%, 1.01 \%$, and $6.53 \%$ for the maximum relative error, minimum relative

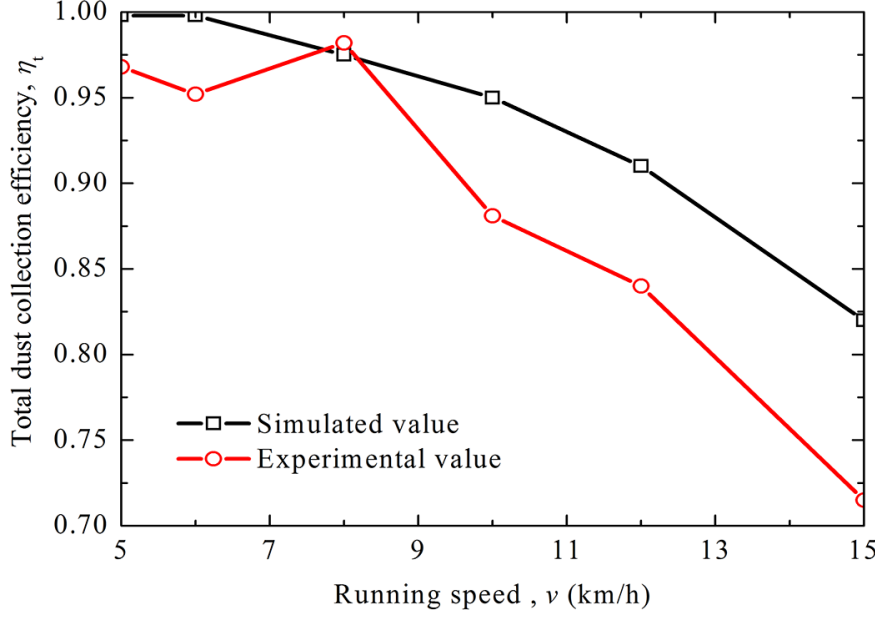

Fig. 17. Comparison of dust collection efficiency associated with different running speeds.

error, and average relative error, respectively. Therefore, CFD-based simulation of the vacuum-blowing cleaning system is feasible. 
The relative error is determined from:

$$
\Delta p=\left|\frac{p_{s}-p_{t}}{p_{t}}\right| \times 100 \%
$$

Where, $\Delta p, p_{\mathrm{s}}$, and $p_{\mathrm{t}}$ are the relative error, simulated value, and measured value, respectively.

\section{Conclusion}

Preliminary theoretical calculations of the structure and vacuum-blowing cleaning system have been performed and the size interference of the vacuum-blowing cleaning system in the assembly of the road sweeper is considered. The suction-inlet diameter, suction-inlet inclination angle, and front baffle inclination angle of the vacuum-blowing cleaning system are selected as the main influence factors, and each factor is evaluated via single-factor effect analysis. The influence of these three structural parameters on the dust collection performance of the vacuum-blowing cleaning system is determined through a uniform optimization design and multiple regression analysis method. In addition, a regression equation considering interaction between the factors is formulated, and the average velocity of the front inlet surface comprising the vacuum-blowing cleaning system is taken as the optimization objective. The results of this study are summarized as follows:

- The suction-inlet diameter, suction-inlet inclination angle, and front baffle inclination angle influence the dust collection performance of the vacuum-blowing cleaning system. Single-factor effect analysis shows that the dust collection performance increases with increasing suction-inlet diameter. Furthermore, the dust collection efficiency increases initially and then decreases when the inclination angle of the inlet or front baffle increases.

- The factors may be written in descending order of their influence on the dust collection efficiency of the vacuumblowing cleaning system, that is, $x_{1}$ (suction-inlet diameter) $>x_{3}$ (inclination angle of front baffle) $>x_{2}$ (inclination angle of suction-inlet).

- The interaction between the suction-inlet diameter and the front baffle inclination angle exerts the most influence on the dust collection efficiency of the vacuum-blowing cleaning system. The interaction between the inclination angle of the suction-inlet and the inclination angle of the front baffle exerts the second-most significant influence. However, the interaction between the suction-inlet diameter and its inclination angle exerts only a weak influence.

- The maximum grade dust collection efficiency is realized under the following conditions: suction-inlet diameter: $200 \mathrm{~mm}$, suction-inlet inclination angle: $110^{\circ}$, and front baffle inclination angle: $105^{\circ}$. The total dust collection efficiency of the vacuum-blowing cleaning system before and after optimization is $89.92 \%$ and $96.10 \%$ (i.e., $6.18 \%$ higher), respectively. Experimental verification revealed an average relative error of $6.53 \%$.
This research was supported by the China Postdoctoral Science Foundation(2020M672084, 2018M631167); Liaoning Key Laboratory of Chemical Additive Synthesis and Separation Open Foundation (ZJKF2005). National Natural Science Foundation of China (21706023, 51806114, 51874187). Yingkou Science and Technology Bureau of Creation and Innovation for Company and Doctor.

\section{References}

[1] J. Sun, Y.S. Wang, F.K. Wu, G.Q. Tang, L.L. Wang, Y.H. Wang, Y. Yang, Vertical characteristics of VOCs in the lower troposphere over the North China Plain during pollution periods, Environ. Pollut. 236, 907-915 (2018)

[2] R.J. Zhang, M.X. Wang, X.Y. Zhang, G.H. Zhu, Analysis on the chemical and physical properties of particles in a dust storm in spring in Beijing, Powder Technol. 137, 77-82 (2003)

[3] X.G. Cheng, B. Yang, G.Q. Liu, T. Olofsson, H.B. Li, A total bounded variation approach to low visibility estimation on expressways, Sensors 18, 18 (2018)

[4] G.-l. Liu, W.-b. Huang, P.-w. Chen, S.-h. Xue, Research on fog and haze purification technology for highways, J. Highw. Transp. Res. Dev., Engl. Ed. (USA) 10, 102-110 (2016)

[5] C.J. Liu, C.Y. Liu, N.T. Mong, C.C.K. Chou, Spatial correlation of satellite-derived PM2.5 with hospital admissions for respiratory diseases, Remote Sens. 8, 15 (2016)

[6] J. Vempilly, B. Abejie, V. Diep, M. Gushiken, M. Rawat, T.R. Tyner, The synergetic effect of ambient PM2.5 exposure and rhinovirus infection in airway dysfunction in asthma: A pilot observational study from the central valley of california, Exp. Lung Res. 39, 434-440 (2013)

[7] Y. Chen, G.W.K. Wong, J. Li, Environmental exposure and genetic predisposition as risk factors for asthma in China, Allergy Asthma Immunol. Res. 8, 92-100 (2016)

[8] W.L. Zijlema, K. Wolf, R. Emeny, K.H. Ladwig, A. Peters, H. Kongsgard, K. Hveem, K. Kvaloy, T. Yli-Tuomi, T. Partonen, T. Lanki, M. Eeftens, K. de Hoogh, B. Brunekreef, R.P. Stolk, J.G.M. Rosmalen, The association of air pollution and depressed mood in 70, 928 individuals from four European cohorts, Int. J. Hyg. Environ. Health. 219, 212-219 (2016)

[9] Q. Wang, Z.M. Yang, Does chronic disease influence susceptibility to the effects of air pollution on depressive symptoms in China?, Int. J. Ment. Health Syst. 12, 12 (2018)

[10] Y.S. Song, X.K. Wang, B.A. Maher, F. Li, C.Q. Xu, X.S. Liu, X. Sun, Z.Y. Zhang, The spatial-temporal characteristics and health impacts of ambient fine particulate matter in China, J. Clean Prod. 112, 1312-1318 (2016)

[11] C.I. Falcon-Rodriguez, A.R. Osornio-Vargas, I. Sada-Ovalle, P. Segura-Medina, Aeroparticles, Composition, and Lung Diseases, Front. Immunol. 7, 9 (2016)

[12] X.B. Deng, F. Zhang, L.J. Wang, W. Rui, F. Long, Y. Zhao, D.L. Chen, W.J. Ding, Airborne fine particulate matter induces multiple cell death pathways in human lung epithelial cells, Apoptosis 19, 1099-1112 (2014)

[13] S. Walter, S. Ulli-Beer, A. Wokaun, Assessing customer preferences for hydrogen-powered street sweepers: a choice experiment, Int. J. Hydrog. Energy 37, 12003-12014 (2012) 
[14] A. Karanasiou, F. Amato, T. Moreno, J. Lumbreras, R. Borge, C. Linares, E. Boldo, A. Alastuey, X. Querol, Road dust emission sources and assessment of street washing effect, Aerosol Air Qual. Res. 14, 734-743 (2014)

[15] J. Vaze, F.H.S. Chiew, Experimental study of pollutant accumulation on an urban road surface, Urban Water 4, 379-389 (2000)

[16] Z.J. Chen, W.U. Xiao-Yuan, X.U. Guang-Pu, A.Q. Wang, Experimental study on suction mouth of vacuum sweeper, J. Tongji Univ. 12, 1483-1485 (2001)

[17] Y. Xu, X.-f. Li, T.-y. Xiao, G.-y. Zeng, Research on applications of computational dynamic fluid in the simulation analysis of highway mechanical sweeper, J. Syst. Simul. 16, 270-273 (2004)

[18] Y. Zeng, T. Xiao, X. Li, Y. Xu, Simulation for the design and optimization of dust collection box of highway sweeper, Comput. Simul. 21, 157 (2004)

[19] C.-z. Yang, Y.-c. Zhang, Z.-j. Ouyang, J. Zhang, S.-z. Yang, R.-Z. Zhang, Parametric design of dust collection port of vacuum sweeper based on flow simulation, J. Cent. South Univ. Sci. Technol. 43, 3704-3709 (2012)

[20] G.-y. Zeng, X.-f. Li, T.-y. Xiao, Y. Xu, Simulation for the design of dust suction system of highway sweeper, J. Syst. Simul. 16, 2770-2773 (2004)

[21] Y.C. Zhang, C.Z. Yang, C. Baker, M. Chen, X. Zou, W.L. Dai, Effects of expanding zone parameters of vacuum dust suction mouth on flow simulation results, J. Cent. South Univ. 21, 2547-2552 (2014)

[22] B. Wu, J. Men, J. Chen, Numerical study on particle removal performance of pickup head for a street vacuum sweeper, Powder Technol. 200, 16-24 (2010)

[23] Y. Xi, Y. Dai, X.l. Zhang, X. Zhang, Prediction of particlecollection efficiency for vacuum-blowing cleaning system based on operational conditions, Processes 8, 809 (2020)

[24] D.M. Murphy, M. Parker, N.P. Sullivan, The interplay of heat transfer and endothermic chemistry within a ceramic microchannel reactor, J. Therm. Sci. Eng. Appl. 6, 7 (2014)

[25] G.D. Wehinger, F. Klippel, M. Kraume, Modeling pore processes for particle-resolved CFD simulations of catalytic fixed-bed reactors, Comput. Chem. Eng. 101, 11-22 (2017)

[26] B. Wu, I. Men, I. Chen, Improving the design of a pickup head for particle removal using computational fluid dynamics, Proc. Inst. Mech. Eng. Part C-J. Eng. Mech. Eng. Sci. 225, 939-948 (2011)

[27] E.F. Barrera, F.A. Aguirre, S. Vargas, E.D. Martínez, Influencia del Y Plus en el Valor del Esfuerzo Cortante de Pared a través Simulaciones empleando Dinámica Computacional de Fluidos, Inf. Tecnol. 29, 291-302 (2018)

[28] X.L. Zhang, Y.L. Zhang, Z.M. Liu, J. Liu, Analysis of heat transfer and flow characteristics in typical cambered ducts, Int. J. Therm. Sci. 150, 14 (2020)
[29] B.F. Wu, J.L. Men, J. Chen, Numerical investigation of dusts removal from tramway surface by street vacuum sweeper, in: Z. Cao, Y.H. He, L. Sun, X.Q. Cao (Eds.), Application of Chemical Engineering, Pts 1-3 Trans Tech Publications Ltd., Durnten- Zurich, 2011, pp. 1619-1622

[30] J.R. Berg, H.M. Soliman, S.J. Ormiston, Effective cooling of stacked heat-generating bodies in a large room: Comparison between floor and side-wall air injection, Int. J. Therm. Sci. 47, 787-799 (2008)

[31] F. Han, E. Ganju, R. Salgado, M. Prezzi, Effects of interface roughness, particle geometry, and gradation on the sandsteel interface friction angle, J. Geotech. Geoenviron. Eng. 144, 12 (2018)

[32] F.L. Zhu, G.Z. Zhang, J. Chen, Flow field analysis and structure optimization of vacuum sweeper suction mouth, Mach. Des. Manuf. 11, 50-52 (2008)

[33] T.G. Chuah, J. Gimbun, T.S.Y. Choong, A CFD study of the effect of cone dimensions on sampling aerocyclones performance and hydrodynamics, Powder Technol. 162, 126-132 (2006)

[34] M. Jain, M.R. Paranandi, D. Roush, K. Goklen, W.J. Kelly, Using CFD to understand how flow patterns affect retention of cell-sized particles in a tubular bowl centrifuge, Ind. Eng. Chem. Res. 44, 7876-7884 (2005)

[35] G. Genin, J.L. Houzelot, A. Kotro, G. Martin, New methods for measurement of residence time distributions of transported solid particles, Chem. Eng. J. 81, 301-306 (2001)

[36] H. Zhao, J. Li, Q.S. Zhu, H.Z. Li, Modulating the mean residence time difference of wide-size particles in a fluidized bed, Chin. J. Chem. Eng. 26, 238-244 (2018)

[37] B.B. Yang, K.R. Swartzel, Particle residence time distributions in 2-phase flow in straight round conduit, J. Food Sci. 57, 497-502 (1992)

[38] K. Fang, F.J. Hickernell, The uniform design and its applications, Hong Kong Baptist University, Department of Mathematics, 1995

[39] Z. Xu, T. Dong, J. Kang, X. Zhao, H. Wang, G. Li, Parameters optimizing of NiCr-Cr3C2 coating deposited by supersonic plasma spraying based on uniform design, J. Mech. Eng. (China) 50, 43-49 (2014)

[40] H.L. Ping, G.H. Xu, S.F. Wu, System optimization of cyclohexane dehydrogenation under multiphase reaction conditions using the uniform design method, Int. J. Hydrog. Energy 40, 15923-15932 (2015)

[41] J. Yang, L. Li, L.Y. Yang, J.Q. Li, Uniform design for the parameters optimization of pin-fins channel heat sink, Appl. Therm. Eng. 120, 289-297 (2017)

[42] F. Kaitai, Uniform design and uniform design table, Science Publication, 1994

[43] J.B. Sim, U.H. Yeo, G.H. Jung, S.B. Park, G.N. Bae, S.J. Yook, Enhancement of louver dust collector efficiency using modified dust container, Powder Technol. 325, 69-77 (2018) 